\title{
Etude des régulateurs de vitesse des turbines hydrauliques au point de vue de leur influence sur le rendement global des installations
}

Par CAYERE. Ingénieur A. M. et I. E. G., Ingínieur cn chef aux Etablissements Neyret-Beylier-Piccard-Piclet.

La lillerature technique sur les régulateurs des turbines hydrauliques comporte déjà de nombrénses études et, rien qu'en France, on peut citer de les remarquables travaux de MM. Leauti, Rateau. Ronlin, Barbillion, Gariel, etc..., qui s'occuperrent successivement du probleme délicat et passionnant de la régulation de la vitesse des turbines.

Dans ces diverses études, bien que le problème ait été abordé duns toule son amplaur, c'est surtout le rôle fondamental du régulateur qui a 'té átudié, ce róle étant de maintenir une vitesse régulière de la turbine malgré lés variations de la puissance qui lui est demandée.

A côté de ce role fondamental, lé régulateur est investi d'une fonction accessoire, mais nullement négligeable. Il est chargé, en effet, de réglér la puissance de la turbine lorsqu'elle est couplée électriquement ou méchniquement avec d'autres machines, ot de faire dévolopper par cette tarbine une fraction convenable de la puissance totale demandée à l'ensemble.

C'est ce problème secondaire, en apparence seulement, qui a été aborté sommairement dans les trawaux antérteurs cités plus haut, que nous voudrions approfondir quelque peu au cours de la présente note.

L'importance pratique de ce problème est indispensable ; les régulateurs des turbines d'un réseau étant chargés de répartir entre les diverses usines la demande de puissance de l'ensemble du réseau, il importe qu'ils remplissent ce rôle de la meilleure façon possible, pour assurer le maximum d'utilisation de l'énergie hydraulique des diverses usines.

Dans cette note, nous exuminerons successivement trois éléments distincts du problème:

$1^{\circ}$ Le rôle des régulateurs dans la répartition oplimum de la charge d'un réseau entre les diverses usines. de réserve.

20 Le rôle des régulateurs dans l'utilisation optimum de l'́nergie hydraulique des usines n'ayant pas lique.

$3^{\circ}$ Le róle des rẹ́gulateurs dans l'utilisation optimum de l'énergie des usines ayant une réserve hydrau-

I. - Rôle des Régulateurs dans la répartition optimum de la charge d'un réseau entre les différentes usines

Rappelons tout d'abord sommairement par quel mécanisme les régulateurs de vitesse assurent la répartition de la charge entre les diverses machines d'un réseau.

Le régulateur de vitesse d'un groupe électrogène a pour but, en première analyse, de régulariser la vitesse de ce groupe. Quand on cxamine le problème de plus près, on arrive à une définition plus précise : "Le régulateur d'un groupe électrogène a pour mission d'établir entre la vitesse de régime et la puissance une relation (1) (p) appelée caractéristique de statisme ». Cette caractéristique $s$, représentée par la fig. 1, doit être tombante vers les fortes charges pour assurer la stabilité de la répartition des charges entre des groupes couplés en parallèle, soit électriquement, soit mécaniquement, et astreints ainsi à tourner à des vitesses rigoureusement correspondantes.
Fig. 1.

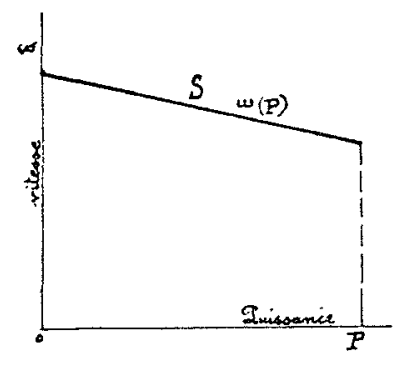

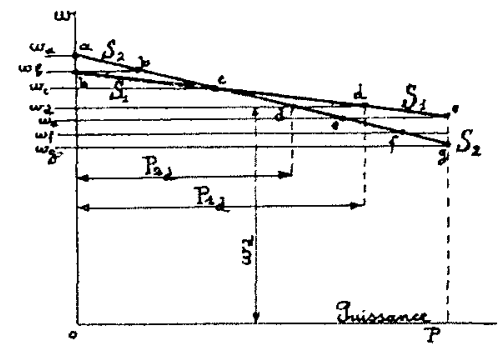

Fig. 2
Si nous considérons deux groupes couplés en parallèle et si nous représentons leurs deux caractéristiques de statisme $S_{1}$ et $S_{2}$, (fig. 2), nous pouvons étudier facilement ce qui se passe lorsque la charge totale varie.

Considérons une vitesse de régine a imposée aux deux groupes par la charge du réseau. Nous voyous, d'après la fíg. 2 , que les deux groupes développent, pout celte vilesse, l'un une puissance
$P_{1 d}$, l'autre une puissance $P_{2 d}$; la puissance totale du résean est alors :

$$
P_{d}=P_{1 d}+P_{2 d}
$$

Pour cette puissance totale du réseau, nous connaissons done toutes les conditions de marche des deux groupes, leur vitessc commune $\omega_{d}$ et leurs puissances respectives $P_{1}$ d et $P_{2 d}$.

En opérant de mème pour d'autres vitesses, nous pouvons élablir le graphique de la figure 3 qui donne, pour une charge quelconque du réseau, la vitesse commune des groupes, ou plus généralement la fréquence du réseau, et la répartition de la charge entre les divers groupes.

Si nous supposons que la charge totale augmente de $\triangle P$, nous voyons que cette augmentation de charge se répartıt sur les deux groupes en deux augmentations $\lrcorner \mathrm{P}_{1}$ et $\lrcorner \mathrm{P}_{2}$, la plus petite élant celle du groupe 2 dont la caractéristique de statisme $S_{2}$ est la plus tornbante.

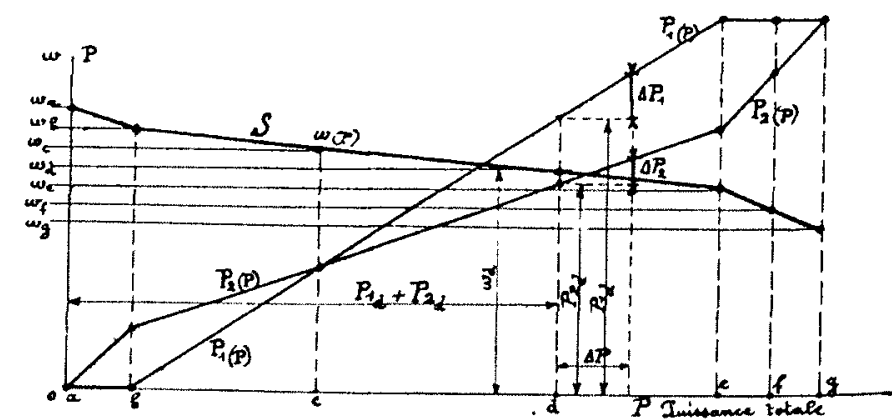

Fig. 3

L'écart entre les vitesses de régime extrêne définies par la caractéristique de statisme s'appelle Décrément de Stalisme ou encore Décompensation, et nous pouvons exprimer, le résultat de notre etude de la figure 3 , en énongant ce lemme :

Les variations de charge d'un réseau se répartissent entre les 
dwers groupes générateurs de ce réseau en raison inverse de leur décrément de statisme.

Nous pouvons donc, en faisant varier le décrément de slalisme d'un groupe, comme cela est prévu dans les régulateurs modernes, faire varier l'aplitude de ce groupe a participer aux variations de charge du réseau; un statisme faible lui faisant prendre une part importante de ces variations de charge, un statisme imporlant lii laissant au contraire une charge à peu près constante.

Or, dans les diverses usines d'un réseau, on peut considérel deux catégories d'usines hydrauliques. Les premières sont les usines sans accumulation dans lesquelles on dispose d'une puissance hydraulique indépendante de la charge du réseau, et fui demandc à être utilisée intégralement sous peine d'être perdue. Les yroupes de ces usines devront donc avoir un décrément de stalisme important (5 à $6 \%$ ) afin de marcher à puissance constante, rette puissance étant d'autre part ajustée d'après la puissance hydraulique de la chute.

Kola.- Nous croyons devoir faire remarquer que la présente itude envisage: seulement la répartition des variations de charge curégime établi. Pendant les perturbations en effet, la répartition des variations de charge est déterminée par d'autres éléments, an particulier l'incrtie des groupes et les durées de manceuvre des régulateurs : nous n'aborderons pas cette question spéciale, nous contentant de la signaler.

\section{II. - Rôle des Régulateurs dans l'utilisation optimum de l'énergie hydraulique des usines n'ayant pas de réserve}

Dans cette catégorie dusines, qui comprend la plupart des usines de basses chutes et un grand nombre d'usines de chutes moyennes, on dispose d'un débit qui doit ètre entièrement utilisé dans les turbines sous peine d'ètre perdu.

Ce debit peut être variable au cours de la journée, pour diverses canses dont les principales sont :

10 Les variations de débit des usines situées en amont et possétant une réserve, usines qui sont amenées à faire les pointes ct fonctionnent ainsi à charge variable ;

$2^{\circ}$ Les diverses servitudes d'irrigation ou de navigation, qui peuvent absorber un débit variable au cours de la journée;

$3^{0}$ Les variations naturelles du débit des aftluents alimentés par des glaciers dont le débit diurne est sensiblement supérieur au débil nocturne ;

Le criterium du fonctionnement optimum d'une telle usine est ividemment d'utiliser toul le débit dans les turbines et d'utiliser lout le débit avec le maximum de chute.

Cette double condition se trouve remplie si le niveau amont est maintenu dans le voisinage du niveau du déversoir du barrage, légèrement au-dessous pour eviter toute perte d'eau par re déversoir, mais très peu au-dessous pour ne pas perdre une fraction de la chute.

Il faut donc constammenl régler le débit absorbé et la puissance developpée par les lurbines pour maintenir le niveau amont: it cette cote, el cela, d'une part malgré les variations du débit de la rivière et d'autre part malgré les variations de la puissance demandée par le réseau.

Le maintien de ce niveau optiunum peut ètre assuré par le personnel de l'usine intervenant a chaque instant pour régler convenablement la puissance débitéc par les lurbines.

On conçoit facilement que ce réglage demande de lattention et de l'habileté, et il y a évidemment intérêt à le rendre automatique, Pour cela, il suffit d'adjoindre aux régulateur's de l'usine des organes mettant les mécanismes de répartition des charges sous la dépendance du niveau amont. Les régulateurs de vitesse deviennent ainsi des régulateurs de niveau amont, sans cesser d'ailleurs de jouer leur rôle de régulateurs de vitesse.

Nous allons examiner rapidement les différents types de régulateurs de niveau qui peuvent être adjoints aux régulateurs de vitesse.

On peut considérer deux grandes classes de ces appareils ; ceux à action directe et ceux à action indirecte.

\section{A. - Régulateurs de iiveau a action directe}

Le type le plus simple de ces appareils est celui couramment réalisé dans les basses chutes, lorsque le régulateur se trouve au voisinage immédiat de la chambre d'eau.

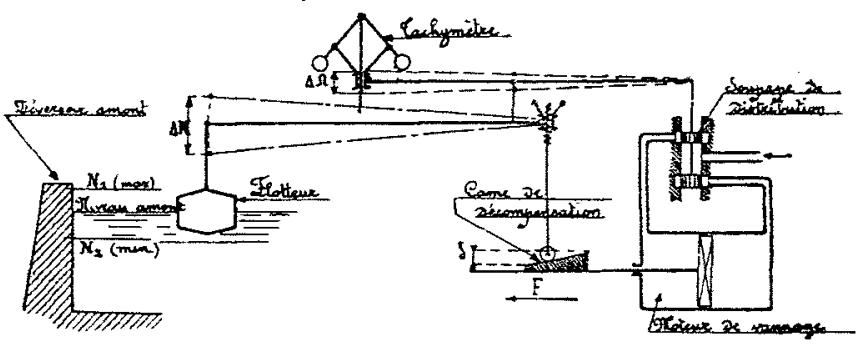

Fig. 4

Un flotteur, installe dans la chambre deau, suit les variations du niveau amont et agit par un système de leviers sur l'organe du régulateur, qui règle la puissance débitée par la turbine sur le réseau (fig. 4).

Le déplacement de cet organe qui, dans la fig. 4, est le point d'articulation de la timonerie du régulateur, se traduit par une translation de la caractéristique de statisme $\mathrm{S}$ de la turbine (fig. 5).

Nous avons représenté dans la fig. 5, d'une part la caractéristique de statisme $\mathrm{S}$ de la turbine, d'autre part la caractéristiquc de statisme $E$ de l'ensemble des autres groupes du réseau. Nous avons supposé, pour simplifier les raisonnements, que cettc caractéristique $\mathrm{E}$ est sensiblement horizontale, c'est-à-dire que l'ensemble des autres groupes du réseau a un statisme faible.

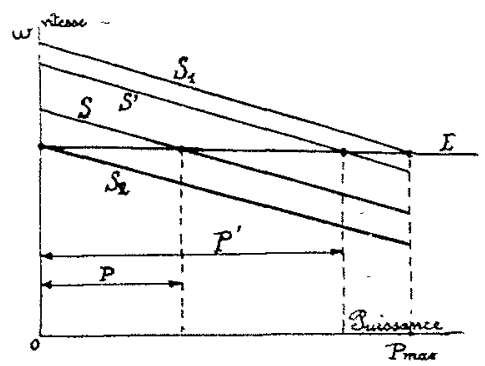

Fig. 5

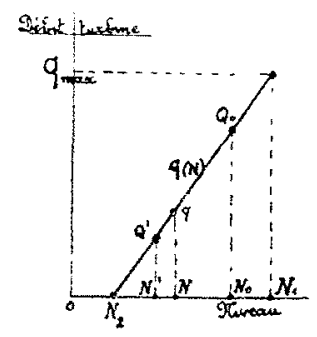

Fig. 6
Avec cette hypothèse simplificatrice, qui revient à dire que la fréquence du réseau est indépendante de la charge, on voit par la figure 5 que la puissance développée par la turbine dépend uniquement de la position de la caractéristique $\mathrm{S}$ de statisme. Pour la position $S_{1}$, correspondant au niveau maximum, la turbine fonctionne à pleine charge; pour la position $\mathrm{S}_{2}$, correspondant au niveau minimum. la turbinc marche à vide.

Le débit de la turbine passe donc de zéro au maximum, quand le niveau passe de $\mathrm{N}_{2}$ à $\mathrm{N}_{1}$ (fig. 6).

Examinons maintenant ce qui se passe lorsqu'il se produit une variation brusque du débit de la rivière. A un instant initial $t_{0}$, (fig. 7), le débit de la rivière est $Q_{0}$, le niveau est $N_{0}$ et le débit de la turbine est $q_{0}=Q_{0}$. 
Supposons que le débit de la riviere diminue brusquement et passe de $Q_{0}$ à $Q^{\prime}$, par suite d'une baisse de charge dans une usine amont par exemple. Le niveau va d'abord baisser à une allure:

$$
\frac{d \mathrm{~N}}{d t}=\frac{Q_{n}-Q^{\prime}}{1 .}
$$

1. étant la surface de la nappe d'eau amont, barrage, canal d'amenée, chambre d'eau.

Cette baisse de niveau agit, par l'intermediaire du flotteur et du régulateur, sur le débit $q$ de la turbinc.

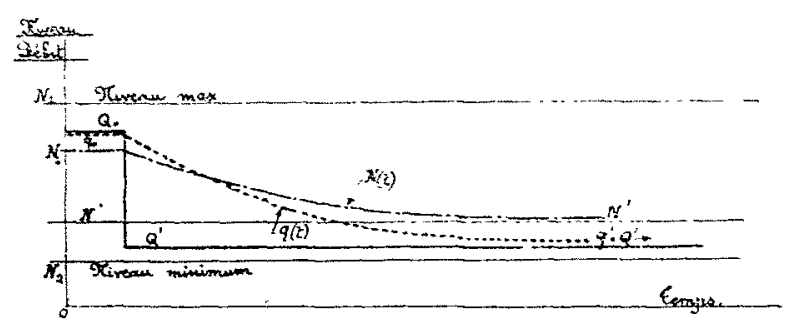

ligi 7

A un instant quelconque, la loi de rariation du niveau amonl est donnée par :

$$
\frac{d N}{d t}=\frac{Q^{\prime}-t}{\Omega}
$$

Prenons comme origine des niveaux le niveau minimum $\mathrm{N}_{2}$, i] vient (voir fig. 6) :

d'où

$$
\eta=q_{\max } \frac{x}{N}
$$

$$
\frac{d N}{d t}=\frac{Q^{\prime}}{Q}-\frac{q \max }{Q}=\frac{N}{N_{1}}
$$

équation qui admet pour solution générale

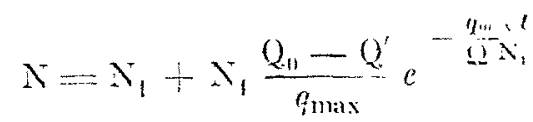

Au temps $t=0$, on a

$$
N=N_{1} \frac{Q^{\prime}}{q_{\max }}+N_{1} \frac{Q_{1}-Q^{\prime}}{q_{\max }}-N_{1} \frac{Q_{01}}{q_{\max }}=N_{1}
$$

Au temps $1=\infty$, on a

$$
X=N_{1} \frac{Q^{\prime}}{q_{\max }}=N^{\prime}
$$

Ce qui montre que le niveau part de la valeur $N_{0}$ correspondant au débit initial, pour suivre une loi exponentielle de variation en fonction du temps et se rapprocher du niveau ${ }^{*}$ ' correspondant au nouveau débit, et l'atteindre au bout d'un temps théoriquement infini, et pratiquement d'autant plus court que $q_{\max }$ est plus grand et que $Q$ et $N_{1}$ sont plus petits.

On voit ainsi que le fonctionnemant d'un régulaleur de niveau it artion directe est tout à fait stable et que le passage d'un rrveau de régime à un autre se fail sans oscillations.

.Il est du reste intéressant de remarquer qu'il y a analogie complète entre les équations de fonctionnement d'un regulateur de niveau à action directe et les équations qui permettent d'étudier, en régime troublé, le fonctionnement d'un groupe électrogène muni d'un régulateur de vilesse à action directe.

La surface $\$$ de la relenue amont tient la place du momenl d'inertie, le niveau joue le rôle de la vitesse, les débits de la rivière et de la turbine jouent le rôle du couple moteur et du couple résistant dans les équations de la régulation de vitesse.
Dans certaines installations, la distance entre la chambre d'ean cl la salle des machines empèche d'utiliser une tringlerie mecanique pour relier le flotteur an régulaleur de vitusse ; on pent alors employer divers systèmes de transmission, par rxemple ha transmission pnemmatique, fig. 8, ot la transmission ilectro-mntmilique, fig. 9.

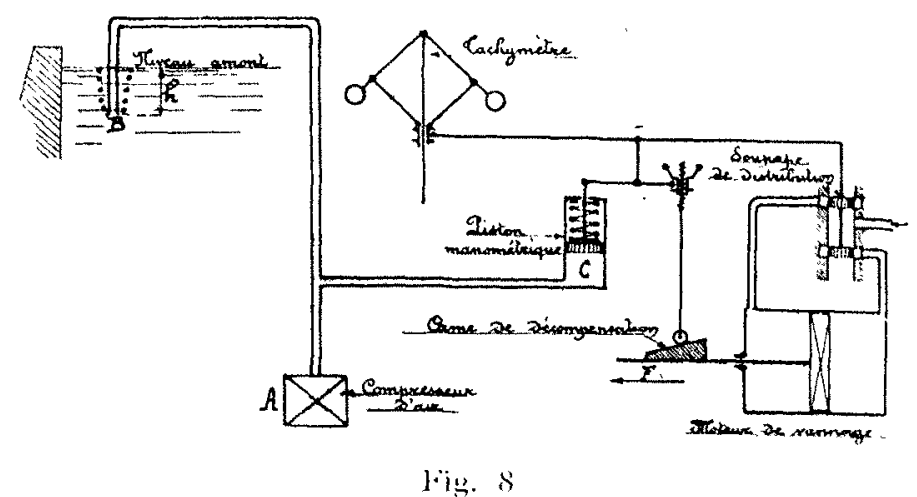

Dans la première, il ny a plus de flottenr; un petit compres. seur d'air A refoule de l'air dans une canalisation dont unte extrémité débouche en $B$ dans la chambre dean, ct l'autre extrimité est reliée en $\mathrm{C}$ à un manomètre agissaul sur lo rẻgulatcur de vitesse, pour déplacer la caractéristigue de statismc.

La pression de l'air dans la canalisation dépend du niveau dan. la chambré deau, car elle est sensiblement égale à la hauleur de l'eau au-dessus du point d'évacuation 13 de la canalisation. loth indications du manometre suivent done exactemenl les rariation du niveau amont, et il y a encore une relation bien définic cult: ce niveau et la position de la caractéristique de statisme du régulateur.

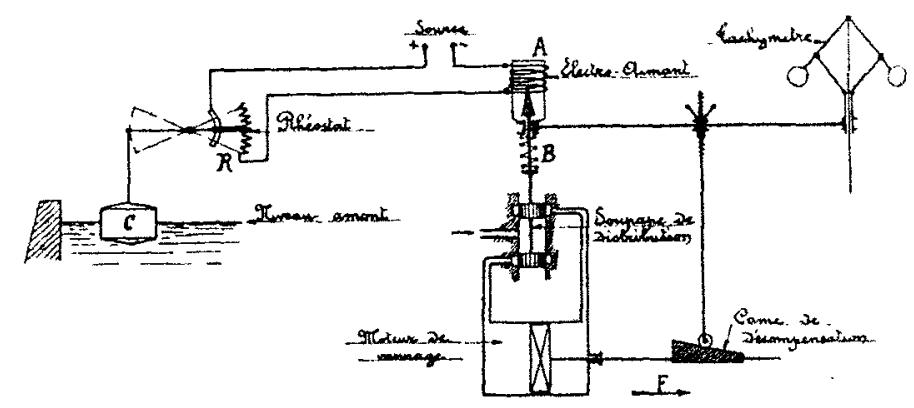

Fis. 9

Dans la Lransnission électromagnélique (fig. 9), le nèue résultat est obtenu en faisant agir un flolleur C place dans la chambre d'eau sur un rhéostat $P$ réglant l'intensité du courant d'ull électro-aimant A monté sur le régulateur. Dans la figure 9, nous avons représenté cel électro sur la tige qui relie la timoneri a la soupape de distribution. Cette lige est extensible, elle tend à se contracter sous l'action de l'électro $A$ el à s'allougur sous l'action d'un ressorl anlagoniste B. Quand le niveau monle, il diminue la résistance du rhéostal, l'intensité dans l'électro augmente, ce qui augmente l'effort de l'électro et raccourcil la lige de soupape. Cetle mancurre a pour effet de remonlor la caractéristirue de slálisme et de faire ouvrir la turbine.

\section{B. - RGGULATEURS DE NIVEAU A ACTION NDDRECTE}

J.es systèmes de régulateurs de niveau à aclion directe que nous venons d'eludier sont avantageux, à cause de leur simplicití et de leur stabilité de fonctionnement.

Ils ont cependant certains inconvénients, généralenent lolerables, mais qui peuvent parfois etre gênants. 
10 Nous avons supposé, dans l'étude précédente, que la fréquence du réseau ne variait pas et nous sommes arrivés à conclure que Ie niveau amont dépendait uniquement du débit.

En rélilé, si la fréquence du réseau varie, le réglage du syslème se trouve modifié. Cela oblige à tolérer des variations do niveau relativement importantes, qui peuvent etre gênantes si l'on a besoin d'un réglage précis du niveau, comme cela peut atre le cas pour certaines servitudes de navigation ou d'irrigation.

$2^{\circ}$ Pour des raisons de construction el de réalisation des mécanismes, le déréglage de la caractéristique de statisme par le flotLeur est généralement limité à une valeur assez faible, 4 à $5 \%$ do la vitesse. Si donc le réseau subit une forte surcharge produisint une haisse de vitesse de plus de $5 \%$, laction du flotteur se trouve insuffisante pour empêcher la turbine d'ouvrir en grand, at il peut en résulter une baisse considérable du niveau amont.

Dans certains cas, cette particularité est avantageuse, le bief amont servant alors de réserve pour passer les fortes pointes, et la turbine absorbant ainsi momentanément un débit supérteur a celui de la rivière, ce qui ne pourrait avoir lieu si le régulateur de niveau restait en action.

Dans dautres cas, au contraire, cette baisse de niveau est inadmissible.

(In peut alors avoir intérêt à employer un régulateur de niveau a action indirecte, plus délical comme fonctionnement, mais pernettant de réaliser un réglage de niveat plus précis et indépendant de la fréquence du nivoau.

Il est facile d'imaginer un régulateur de niveau à action indirecte agissant sur le régulateur de vitesse d'une turbine. La fig. 10 fournit le schéma d'un tel appareil ; le flotteur A ferme, dans un sens ou dans l'autre, le circuit d'un petil moteur qui règle la hauteur du point d'articulation de la limonerie du tachymètre, a par suite la position de la caractéristique de statisme $S$ dans le plan $\mathrm{P}$ (voir fig. 5).

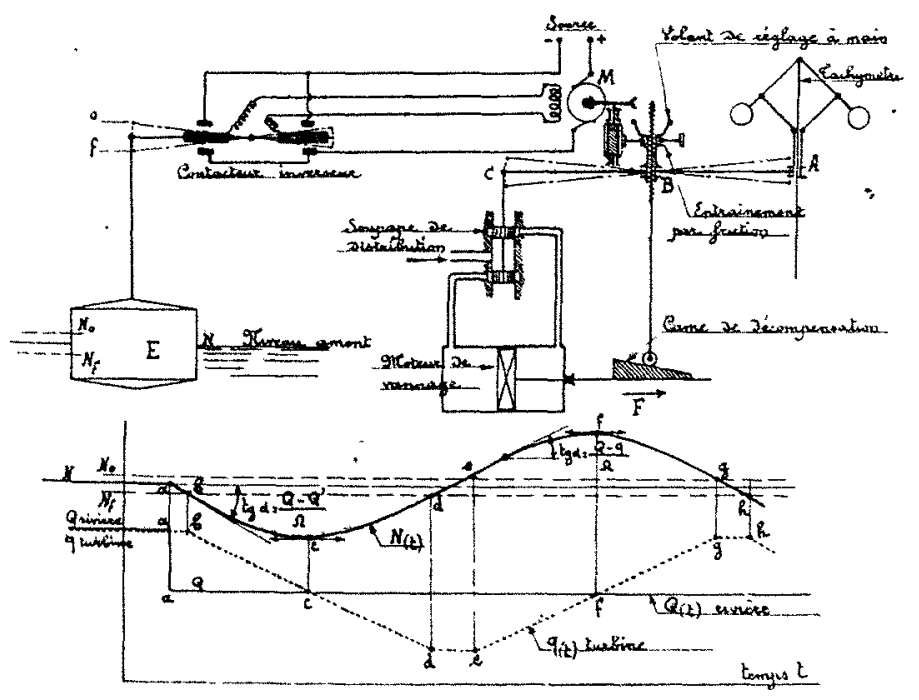

Fig. 10

Fin mison de la constilution du mécanisme, la charge de la turbine est augmentée par lo moteur $M$ quand le niveau est ati-dessous d'une valeur $N_{0}$; elle est diminuée quand il est audessous d'une antre valcur $N_{f}$, qui differce de $N_{0}$ par le jeu qu'on doit laisser dans les intermpteurs, pour permettre les ruptures du courant.

Il semble à première vue qu'un tel dispositif doive maintenir automatiquement le niveau amont entre les limites $\mathrm{N}_{0}$ et $\mathrm{N}_{\mathrm{f}}$.

En réalité, si l'on étudie le problème de plus près, on constale qu'il y a production d'oscillations a longue période, exactement comme dans la régulation indirecte de la vitesse.
La fig. 10 permet de se rendre compte facilement de la production de ces oscillations du niveau amont.

En a, un régime d'équilibre initial Qst troublé pare une.brusque diminution du débit de la rivière; le niveau amont baisse aussitôt à une allure donnée par :

$$
\frac{d N}{d t}-\frac{Q^{\prime}-Q}{\Omega}
$$

(Q étant la surface libre du niveau amont).

En $b$, le niveau a atteint la valeur $N_{f}$, qui actionne le petit moteur $\mathrm{M}$ dans le sens de la réduction de la charge.

Le débit de la turbine diminue donc linéairement suivant une ligne $q(t)$ dont l'inclinaison dépend de la vitesse du moteur $M$, vitesse généralement faible. Pendant ce temps, le niveau continue à baisser, mais de plus en plus lentement, car la manœuvre du moteur M diminue l'écart des débits de la rivière et de la turbine. En $c$, les deux débils sont égaux, le niveau cesse de baisser, mais comme il est trop bas, le moteur $M$ continue à réduire la charge de la turbine et le débit de celle-ci continue à diminuer. Il devient ainsi inférieur au débit cle la rivière, et le niveau amont remonte. En $d$, le niveau repasse à la valeur $N_{f}$ et le moteur $M$ s'arrête, mais comme le débit de la turbine est à ce moment devenu inférieur à celui de la rivière, le niveau continue à monter; en $e$, il atteint la valeur $N_{0}$ et le flotteur envoie le courant dans le moteur, pour augmenter la charge de la turbine, et les mêmes phases se succèdent en sens inverse.

Il est facile d'etudier par l'analyse la loi de variation de $\mathrm{N}$, mais sans entrer dans ce calcul, on peut voir directement, en raison de la symétrie du phénomène, qu'on se retrouve en $h$ dans la même situation qu'en $b$, et qu'ainsi les oscillations du niveau seront indéfinies.

Pour les amortir, il faut employer un artifice, comme dans la régulation indirecte de la vitesse. Cet artifice consiste à avancer l'instant où se produit l'arrêt du moteur $\mathrm{M}$, et à provoquer cet arrêt légèrement après le moment où le niveau passe par un maximum ou un minimum.

Il faut donc réaliser ainsi une sorte d'asservissement, ce terme étant pris dans le sens qu'on lui donne en régulation de vitesse.

On peut envisager un grand nombre de dispositifs d'asservissement. Nous en décrirons seulement deux qui paraissent intéressants par leur simplicité, et surtout par le fait que leur action se détruil automatiquement après la perturbation, ce qui assure le réglage à niveau constant.

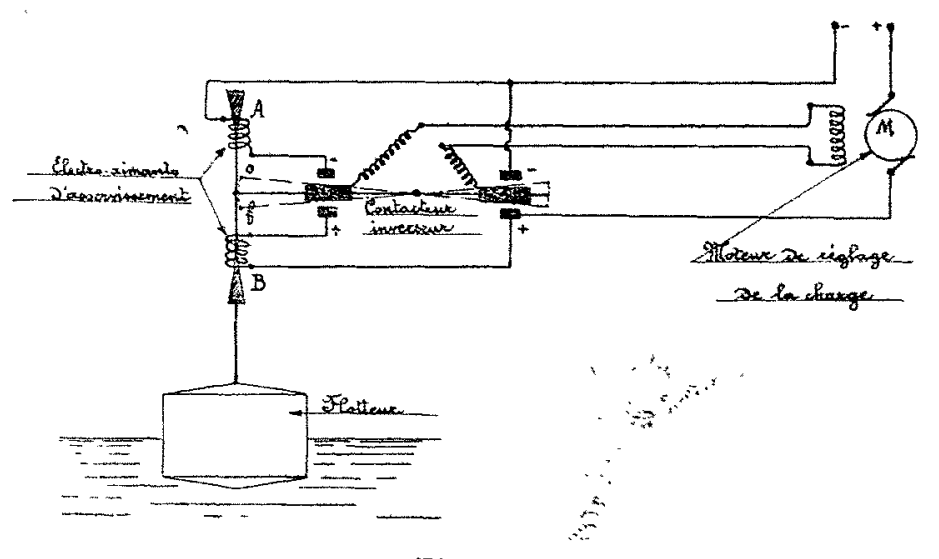

Fig. 11

Dans le premier dispositif, reprěsenté fig. 11, on utilise le principe imaginé par M. J.-L. Routin, et utilisé dans son régulateur électrique. La mise en route du moteur de réglage provoque, sur l'organe qui déclanche cette mise en route, une action antagoniste qui avance l'instant où sera provoqué l'arrêt du moteur de 
réglage. Dans la fig. 11, quand le flotteur monte, il ferme le circuit du moteur $M$ dans lè sens qui provoque l'augmentation de la charge de la turbine; sur le circuit qui est alors parcouru par le courant du moteur, se trouve un électro-aimant A qui attire le flotteur rers le bas, avançant ainsi l'instant où sera provoqué l'arrêt de MI. Quand le niveau baisse, c'est l'électro B qui est alimenté et qui attire le flotteur vers le haut, produisant le même effet stabilisateur.

Dans le deuxième dispositif, représenté fig. 12, la course du flotteur est limitée au minimum nécessaire à la fermeture des circuits du moteur M. Le flotteur comporte un compartiment servant de lest liquide, de poids variable ; co compartiment communique avec l'atmosphère par un large trou et avec la chambre d'eau par un petit orifice. En régime établi, le niveau est le même dans la chambre d'eau et dans le compartiment de lest. Si le niveau monte dans la chambre d'eau, il entraîne d'abord le flotteur, puis, quand celui-ci est arrêté par ses butoirs, le niveau continue à monter dans la chambre d'eau et monte aussi, mais plus lentement, dans le compartiment de lest. Quand le niveau redesc nd, le niveau dans le ccmpartiment. de lest reste audessus du niveau dans la chambre d'eau; le poids du flotteur se trouve ainsi augmenté et l'arrêt du moteur $\mathrm{M}$ a lieu avant que le niveau à la chambre d'eau revienne à la valeur $N_{0}$.

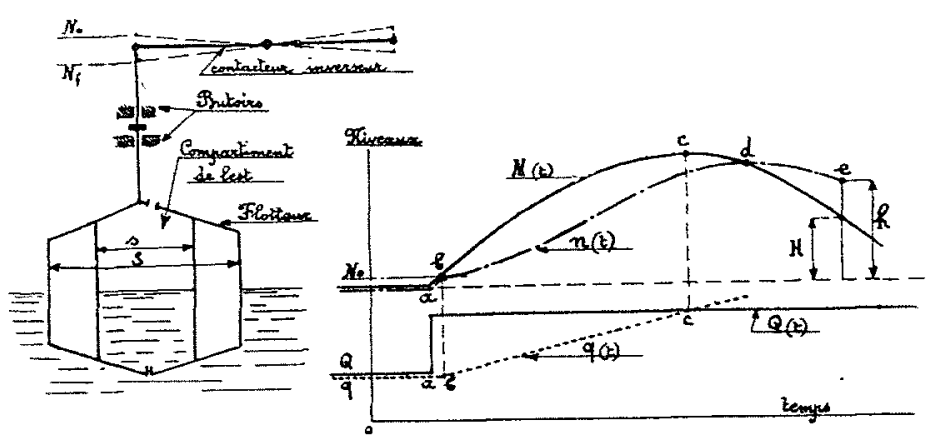

Fig. 12

La figure 12 représente les variations de niveau $\mathrm{N}$ de la chambre d' eau et du niveau $n$ du compartiment de lest. La poussée d'Archimède sur l'ensemble du flotteur est $\mathrm{N} \times \mathrm{S}$; l'augmentation de poids de lest est $n \times s$; le retour du flotteur a donc lieu au moment où on a $\mathrm{H} \times \mathrm{S}=h \times s$, c'est-à-dire au point $e$, à un moment où le niveau redescend, mais n'est pas encore revenu au niveau normal.

Le premier dispositif d'asservissement crée une avance constante du niveau d'arrêt du moteur sur le niveau normal. Il est donc précieux dans les petites perturbations, car son action est indépendante des variations de niveau; le second dispositif d'asservissement a au contraire une action d'autant plus grande que les variations de niveau sont plus importantes. On peut sans difficulté juxtaposer les deux systèmes, afin d'avoir dans toutes les perturbations un amortissement énergique des oscillations du niveau.

\section{III. - Rôle des régulateurs dans l'utilisation optirum de l'énergie des usines ayant une réserve hydraulique}

Ainsi que nous l'avons déjà dit plus haut, ces usines sont appelées à fournir la partie variable de la puissance demandée par le réseau, la partie constante étant fournie par les usines n'ayant pas de réserve.

Il en résulte que les usines qui nous occupent maintenant, fonctionnent à des charges très variables, ces variations pouvant être importantes, rapides, fréquentes et imprévues, ce qui oblige a laisser en service un nombre important de turbines qui, à certaines périodes, travaillent à très faible charge.

Ces conditions d'exploitation, particulièrement marquées danss le cas des réseaux de traction, donnent une importance parliculiere a l'obtention d'un bon rendement aux laibles charges, problème dont on peut obtenir une solution intéressante en dispersant convenablement les régulateurs.

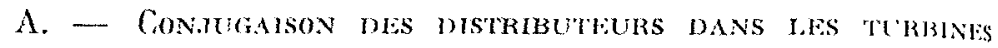
A DISTRIBUTEURS MULTIPLES

Le principe de cette disposition est bien comnu, et il est cmployé depuis longtemps dans les turbines dont les distributeurs sont réglés à la main. Au lieu do laisser tous les distributeurs ouverts à une faible ouverture, pour laquelle ils ont tous un mauvais rendement, il y a avantage a n'en ouvrir que le moins possible et à fermer les autres complètoment. Les distributeurs restant en service fonctionnent ainsi à une ouverture plus grande, et par suite avec un meilleur rendement.

Pour profiter de ces avantage dans les turbines munies de régulateurs, il suffit de lier les distributeurs au régulateur par des mécanismes tels qu'ils assurent automatiquement, à toutes les charges, la conjugaison optimum des injecteurs.

La première idée qui vient à l'esprit pour réaliser cette conjugaison est d'opérer l'ouverture successive des distributeurs, un certain nombre étant complètement ouverts, un l'étant partiellsment, les autres étant complètement fermés, disposition appliquée. autrefois aux turbines Fontaine.

On peut se demander si cette conjugaison correspond à l'obteltion du rendement optimum; nous allons a titre d'exemplr, rechercher systématiquement la meilleure conjugaison des denx injecteurs d'une turbine Pelton double.

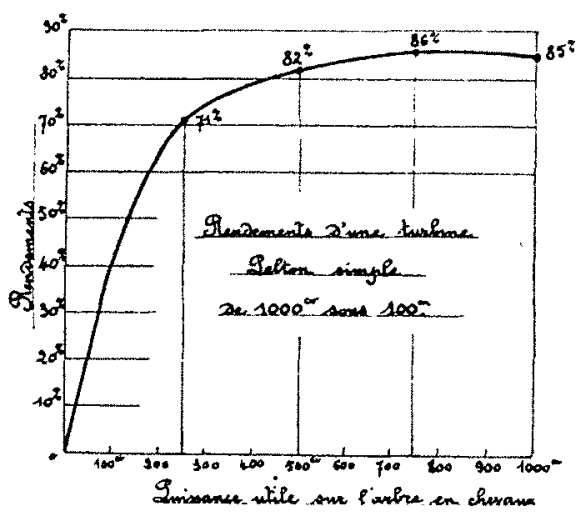

Iig. 13

Considérons la courbe de rendement de la fig. 13 représentant le rendement d'une turbine simple de $100 \mathrm{CV}$. sous $100 \mathrm{~m}$. par exemple, en fonction de la puissance utile sur l'arbre. Proposonsnous de construire une turbine double en ajoutant sur cett: turbine un second jet semblable, agissant sur la même roue, $\mathrm{cl}$ étudions comment il faut conjuguer les deux injecteurs, ponr avoir la meilleure courbe de rendement.

Nous admettrons que le flottement de l'arbre dans ses paliers, ainsi que le brassage de l'air par la roue, absorbe $3 \%$ de la puissance maximum de la turbine simple, perte que nous supposerons constante. Cela nous permet de tracer le graphique de la fig. 1t qui donne le débit de la turbine simple en fonction de la puissance utile sur l'aubage, égale ì la puissance utile sur l'arbre augmentée de 30 chevaux.

Quand nous aurons installé un second jet sur la roue, nous pourrons appliquer à ce jet le graphique de la fig. 14. La puissance totale sur l'arbre est alors égale à : $P=P_{1}+P_{2}-30 \mathrm{CV}$, lo débit total est égal à $Q_{1}+Q_{2}$. 
Nous pouvons construire un graphique simpıe (fig. 15), qui risume les proprietés de la turbine double ainsi constituée, quand on fait varier $Q_{1}$ et $Q_{2}$ de $O$ au max. Nous portons en ordonnée le déhit de l'injecteur 1 , en abcisse le débit de l'injecteur 2 , et nous affectons le point correspondant d'une cote égale à la puissance totale $\mathrm{P}^{\prime}=\mathrm{P}_{1}+\mathrm{P}_{2}-30 \mathrm{CV}$, réalisée avec les deux rébits $Q_{1}$ et (.. Nous pouvons ainsi tracer des courbes d'égale puissance I'. $2^{\circ}$ Lorsqu'un injecteur est insuffisant pour assurer la puissance demandée, on a avantage à ouvrir les deux injecteurs de la même quantité; le graphique montre en effet que, pour une puissance donnée, le minimum de débit correspond à $Q_{1}=Q_{2}$.

Par conséquent, la loi de conjugaison optimum des injecteurs consiste, non pas à les ouvrir successivement, en laissant le premier grand ouvert au-delà de la demi-charge, mais à main-

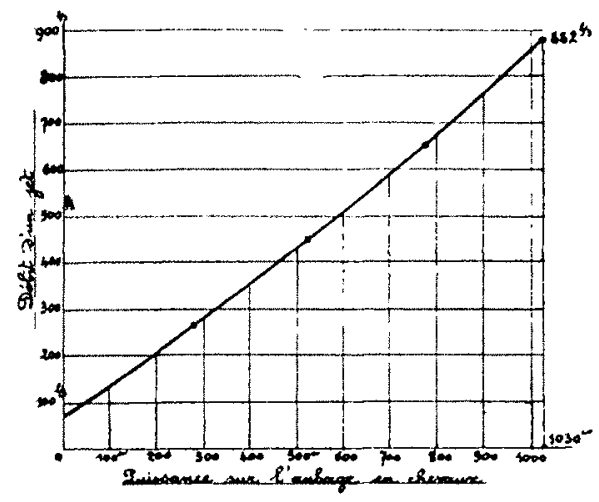

Fig. 11

Nous pouvons également tracer les courbes l'égal débit total $Q=Q_{1}+Q_{2}$. Ces courbes sont dos droites inclinées à $45^{\circ}$. La comparaison du résean de lignes $\mathrm{P}$ totale $=\mathrm{Cl}$ e et $\mathrm{Q}$ total $=\mathrm{Cte}$, nous fixe immédiatement sur la combinaison l'injecteurs qui donne le maximum de rendeinent.

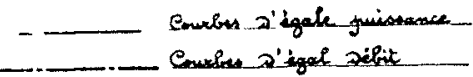

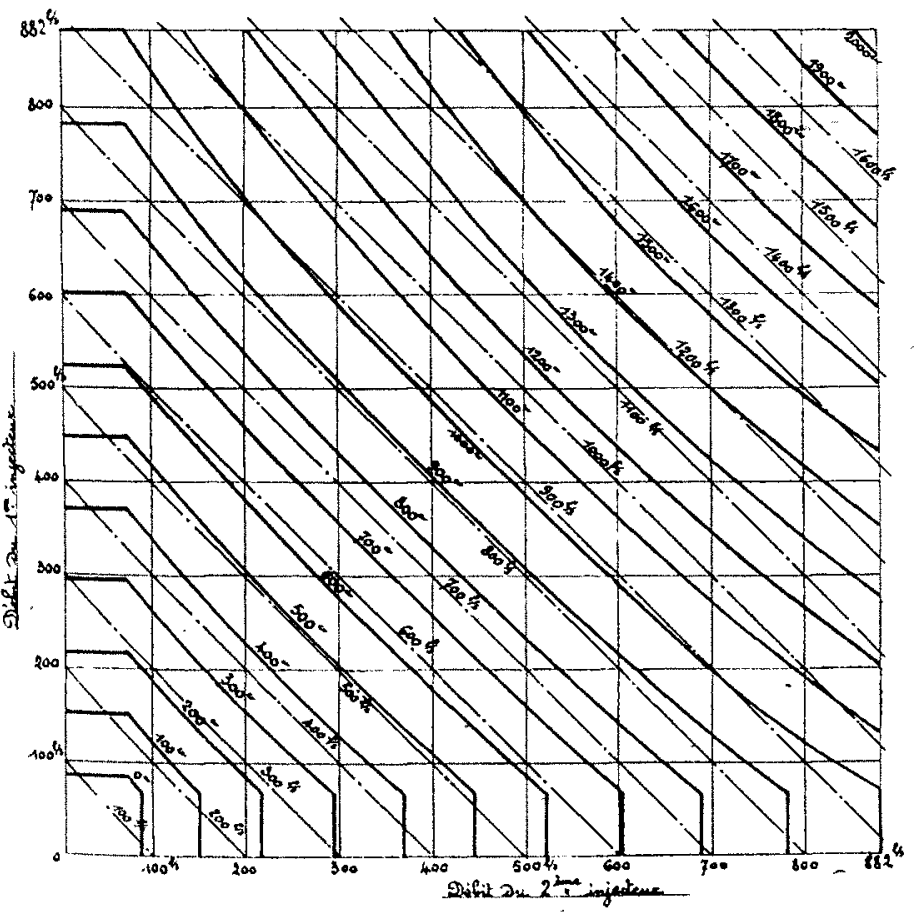

Ijg. 15. - Graphique domnant la puissance utile d'une turbine Pelton double en fonction des débits $Q_{1}$ et $Q_{Q}$ des deux injecteurs.

Ce graphique de la figure 15 conduit, pour une turbine normale, anx conclusions suivantes :

$1^{0}$ Tant qu'un seul injecteur suffit à assurer la puissance demandéc à la turbine, on a avantage à n'ouvrir qu'un injecteur et à laisser l'autre complètement fermé. Le graphique montre en effet que, pour $\mathrm{P}<1000 \mathrm{CV}$, le minimum de débit, pour obtenir une puissance donnée, correspond à : $Q_{1}=0$ ou à $Q_{2}=O$;

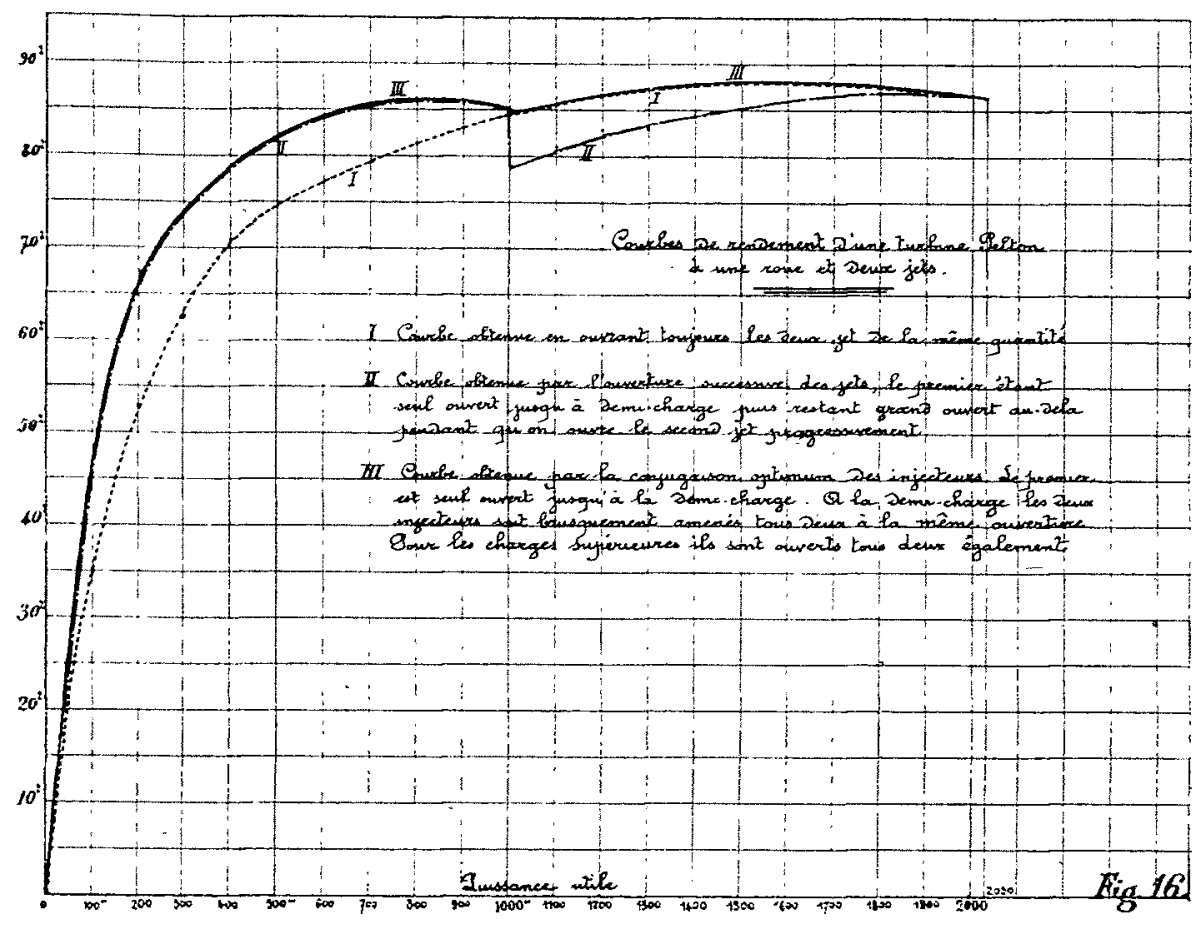

Fig. 16

tenir un injecteur complètement fermé pour toute charge inférieure à la demi-charge, et à ouvrir les deux injecteurs de la même quantité pour toute charge supérieure à la demi-charge.

La fig. 16 représente les courbes de rendement que l'on obtiendrait :

10 En ouvrant constamment les deux injecteurs de la même quantité, comme on le fait ordinairement dans les turbines doubles :

$2^{\circ}$ En ouvrant successivement les deux injecteurs, le premier restant grand ouvert au-dela de la demi-charge ;

$3^{o}$ En utilisant la loi de conjugaison optimum que nous venons d'établir.

La figure 17 montre comment on réalise cette loi de conjugaison optimum des injecteurs, en commandant ceux-ci par le régulateur de la turbine, à l'aide de cames de forme appropriée.

\section{B. - Conjugaison des figllateurs d'une centrale VUE DE L'UTILISATION OPTTMUM DE L'ÉNERGIE}

Le raisonnement que nous avons exposé ci-dessus pour une turbine isolée, s'applique avec la même valeur aux diverses tur. bines d'une centrale, surtout si ces turbines sont des turbines à un seul injecteur, dont le rendement aux faibles charges ne peut pas ètre amélioré par le dispositif décrit plus haut.

Si une centrale comprenant trois turbines doit marcher à $1 / 4$ de charge, tout en conservant les trois machines en service pour parer aux surcharges possibles, il y a intérêt à fermer complètement les distributeurs de deux turbines, qui sont alors entraînées par leurs alternateurs fonctionnant en moteurs synchrones, et à laisser fournir la puissance par la troisieme turbine qui, fonctionnant alors à $3 / 4$ de charge, aura un très bon rendement.

On peut réaliser ce mode de fonctionnement au moyen d'un grand nombre de dispositifs. Nous en décrirons ci-après seulement deux particulièrement simples. 


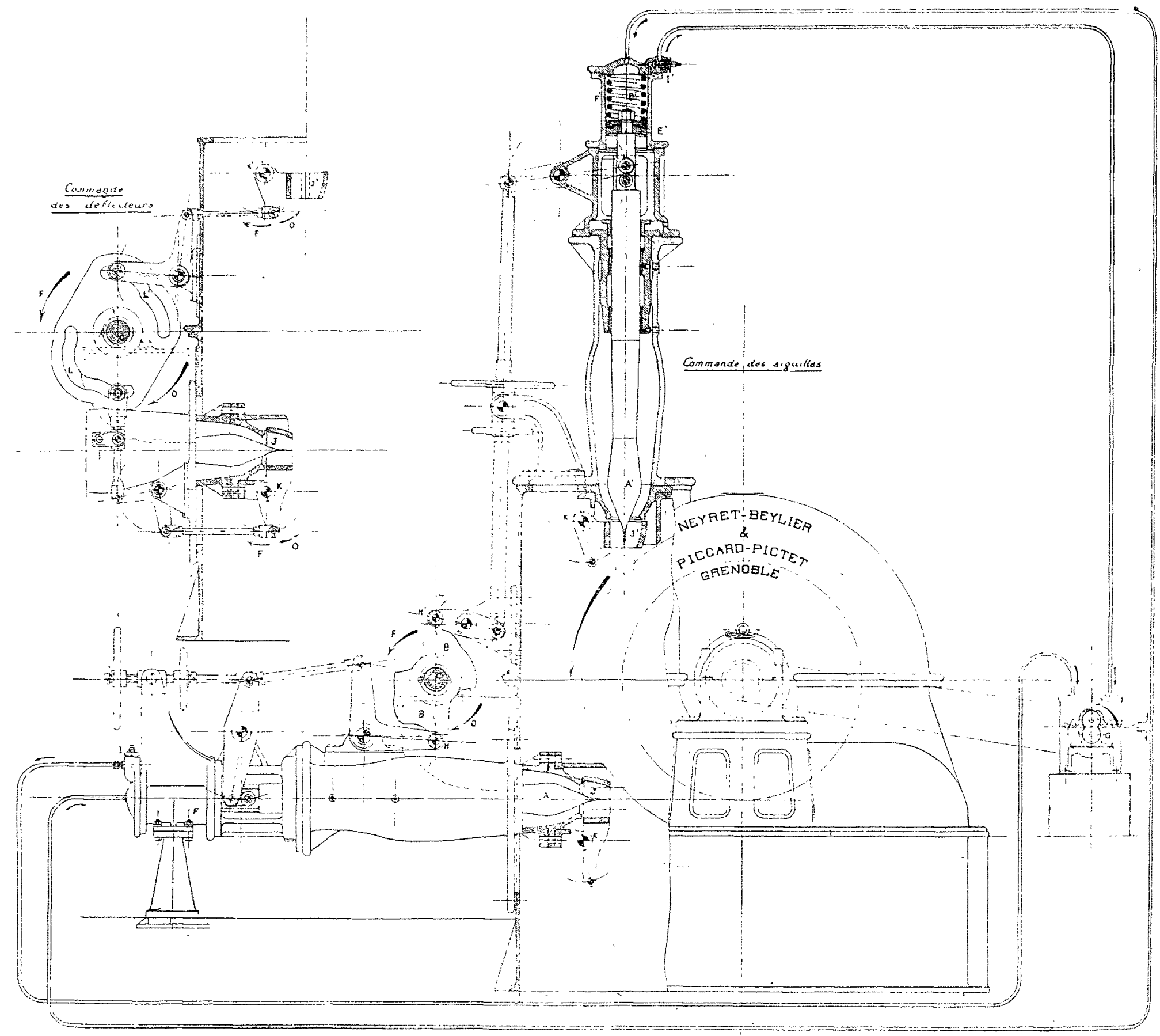

Fig. 17. - Turbine Pelton à deux injecteurs combinés réalisant le maximum de rendement.

Nota. - On peut objecter que la puissance nécessaire à l'entraînement des deux groupes qui tournent à vide est produite par le groupe en charge avec un bon rendement, mais se trouve transmise par les alternateurs marchant en moteurs synchrones à très faible chargë, dont le rendement est par suite très faible, et l'opération peut paraitre peu avantageuse.

En réalité, le rendément à considérer ici pour les moteurs synchrones est excellent ; il n'y a pas à considérer les pertes mécaniques ou par ventilation de l'alternateur, ni les pertes par courants de Foucault, ni les pertes par hystérésis, ni la puissance absorbée par l'excitation, car ces pertes devront toujours être consenties, quel que soit le mode d'entrainement du groupe, puisque celui-ci doit rester couplé en parallèle sur le réseau.

Le rendement à considérer ici concerne donc seulement la transformation de l'énergie wattée reçue par l'alternateur en énergie mécanique transmise à l'arbre de la turbine, et cette transformation, affectée seulement par les pertes par effet Joule et par des pertes secondaires dues à la réaction d'induit, se fait avec un rendement excellent, de l'ordre de 98 à $99 \%$.

En d'autres termes, on doit fournir au groupe, pour tourner à $1^{\circ}$ Une certaine puissance électrique pour l'excitation ;

$2^{\circ}$ Une certaine puissance mécanique pour surmonter les frottements de la ventilation de la turbine, du volant et de l'aitternateur, ainsi que pour compenser les pertes par courant de. Foucault et par hystérésis.

Si l'on emprunte cette puissance mécanique à la chute directement par la turbine, marchant à très faible ouverture, ọn aura un rendement de 25 à $30 \%$ o pour la transformation de l'énergic hydraulique en énergie mécanique. Si l'on emprunte cette puissance mécanique à la chute par l'intermédiaire d'un autre groupe marchant à charge élevéc et entrainant le groupe à vide, par son alternateur fonctionnant en moteur synchrone, on aura :

Rendement de la turbine à charge élevée............ $85 \%$

Rendement de l'alternateur à charge élevée.......... $95 \%$

Rendement de la transformation de l'énergie watlée en énergie mécanique dans la groupe en pleine charge. .. . 98\%

Soit en définitive un rendement de $80 \%$ pour la transformation indirecte de l'énergie hydraulique en énergie mécanique; $c e$ qui justifie bien notre assertion. 
$1^{0}$ Disposition réalisant L'oUverture successive DES TURBINES

II suffit de régler les trois régulateurs pour que leurs caractéristiques de décompensation soient étagées comme le montre la fig. 18.

Si la charge est inféricure au $1 / 3$ de la puissance totale de l'usine, la turbine 1 est. seule chargée, les deux äutres ont leurs distributeurs complètement fermés.

Pour une charge de l'usine comprise entre $1 / 3$ et $2 / 3$ du maxit, mun, la turbine I est ouverte en grand, II est partiellemenonverte, III est complètement fermée.

Enfin, pour une charge de l'usine compris entre $2 / 3$ et $3 / 3^{-}$de la pleine charge, les Lurbines I et II sont ouvertes en grand et li turbine III est ouverte partiellement et assure le réglage.

Cette disposition est évidemment très simple, puisqu'elle ne nécessite l'installation d'aucun organe supplémentaire sur les régulateurs. Par contre, elle a un double inconvénient.
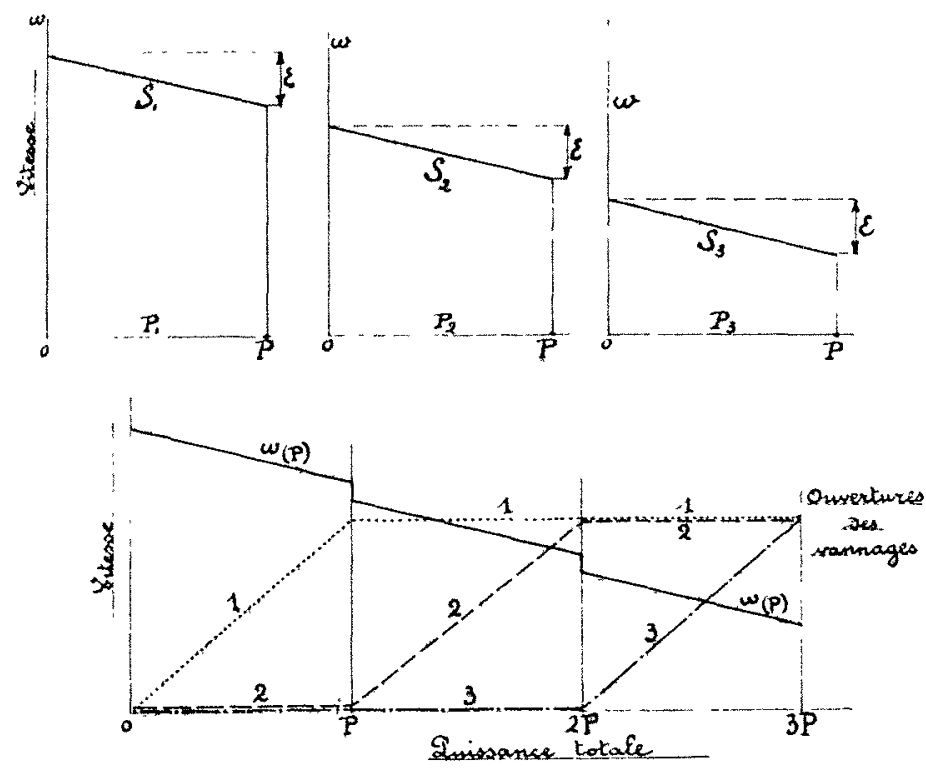

Fig. 18

Tout d'abord, les turbines n'ouvrent pas simultanément, mais seulement l'une après l'autre, ce qui, dans les variations de charge importantes, conduit a une augmentation des écarts de vitesse.

Ensuite, les décréments de statisme des trois régulateurs s'ajoutent, et le décrément total de l'usine, écart entre la marche à vide et la pleine charge des trois groupes, est au moins trois fois plus important que celui d'un groupe seul.

Il y a donc des cas où cette disposition est insuffisante et où il faut employer un autre dispositif.

\section{Mécanisme assurantr la conjugaison optimunt des divers GROUPES D'UNE CENTRALE}

En plus des deux défauts que nous venons de signaler, la disposition précédente avait l'inconvénient, déjà exposé au sujet des turbines à plusieurs injecteurs, de ne pas réaliser la loi de conjugaison cptimum. Cette loi consiste à ouvrir le minimum de turbines nécessaires pour assurer la puissance totale; toutes ces turbines doivent être ouvertes de la mème quantité et les autres doivent etre complétement fermèes.

Le dispositif que nous allons décrire réalise cette loi. Toutes les turbines ont la même caractéristique de décompensation, afị de prendre exactement des charges égales, si on laisse les régulateurs dans leur etat normal.

Les régulatours sont de plus munis d'un dispositif permetitant d'abaisser leurs caractéristiques de décompensation, parallelement d̀ ellemmême et d'une quantité légèrement supérieure au décrément de statisme (fig. 19).
Ce dispositif consiste par exemple en un électro-aimant monté dans la tige de commande de la soupape de distribution, et permettant de modifier la longueur de cette tige d'une quantité déterminée.
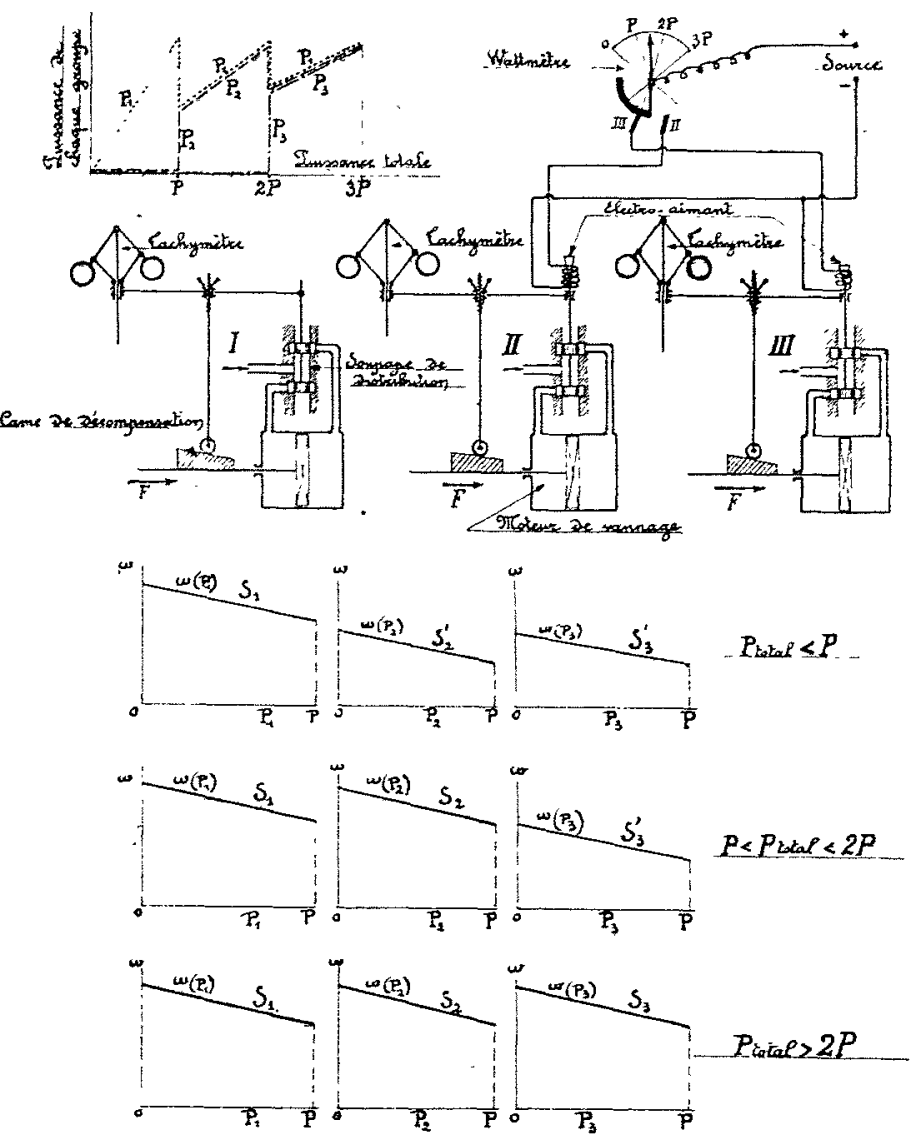

Fig. 19. - Dispositif assurant la conjugaison optimum des divers groupes d'une usine génératrice.

Les circuits des électros sont ouverts ou fermés par un wattmètre indiquant la puissance totale de la centrale.

Quand la puissance demandée à l'usine est inféricure à $1 / 3$ du maximum, le wattmètre envoie du courant dans les électros II et III et allonge les tiges de commande des soupapes de distribution. Les caractéristiques de décompensation de II et III sont ainsi en $S_{2}{ }_{2}$ et $S_{3}$, et les deux turbines II et III sont complètement fermées, le réglage étant assuré par la turbine $I$.

Quand la puissance est comprise entre $1 / 3$ et $2 / 3$ du maximum, le courant n'est plus envoyé que dans l'électro III et la turbine III est complètement fermée. Les turbines I et II se partagent alors également la charge à foumir.

Enfin. quand la puissance est comprise entre $2 / 3$ et $3 / 3$ du maximum, le courant est coupé dans tous les électros et les trois turbines se trouvant placées dans des conditions identiques se partagent également la charge.

On voit que, dans ce dispositif, le décrément de statisme de l'usine est juste égal à celui d'un groupe. De plus, en cas de charge brusque, le wattmètre libère tous les électros et les trois turbines mancuvrent simuitanément. Le second dispositif décrit n'a donc aucun des inconvénients reprochés au premier.

ETUde de L'APPLICATION DE LA CONJUGaison DES GRoupes D'UNE CENTRALE A UN CAS CONCRET

Afin de préciser l'intérèt pratique de ce qui précède, nous allons étudier dans un cas concret, le gain de rendement qui pourrait être obtenu par une conjugaison convenable des groupes d'une centrale

Nous considérerons, pour fixer les idées, l'usine d'Eget des Chemins de fer du Midi, et nous supposerons qu'a une périoac 
déterminée, les nécessités d'exploitation du réseau de traction obligent à laisser en service trois turbines de $5.000 \mathrm{CV}$. pour parer aux pointes, alors que la puissance moyenne demandée est beaucoup moindre, 4.000 à 5.000 chevaux par exemple.

Les caractéristiques de puissance, de débit et de rendement sonl données par la fig. 20 qui résume les essais qui ont été effectués sur les turbines d'Eget (Foir D. Eydoux et P. T eboucher, R. G. E, juillet 1920).

Ces résultats sont ceux mesurés, sans aucune correction rame-

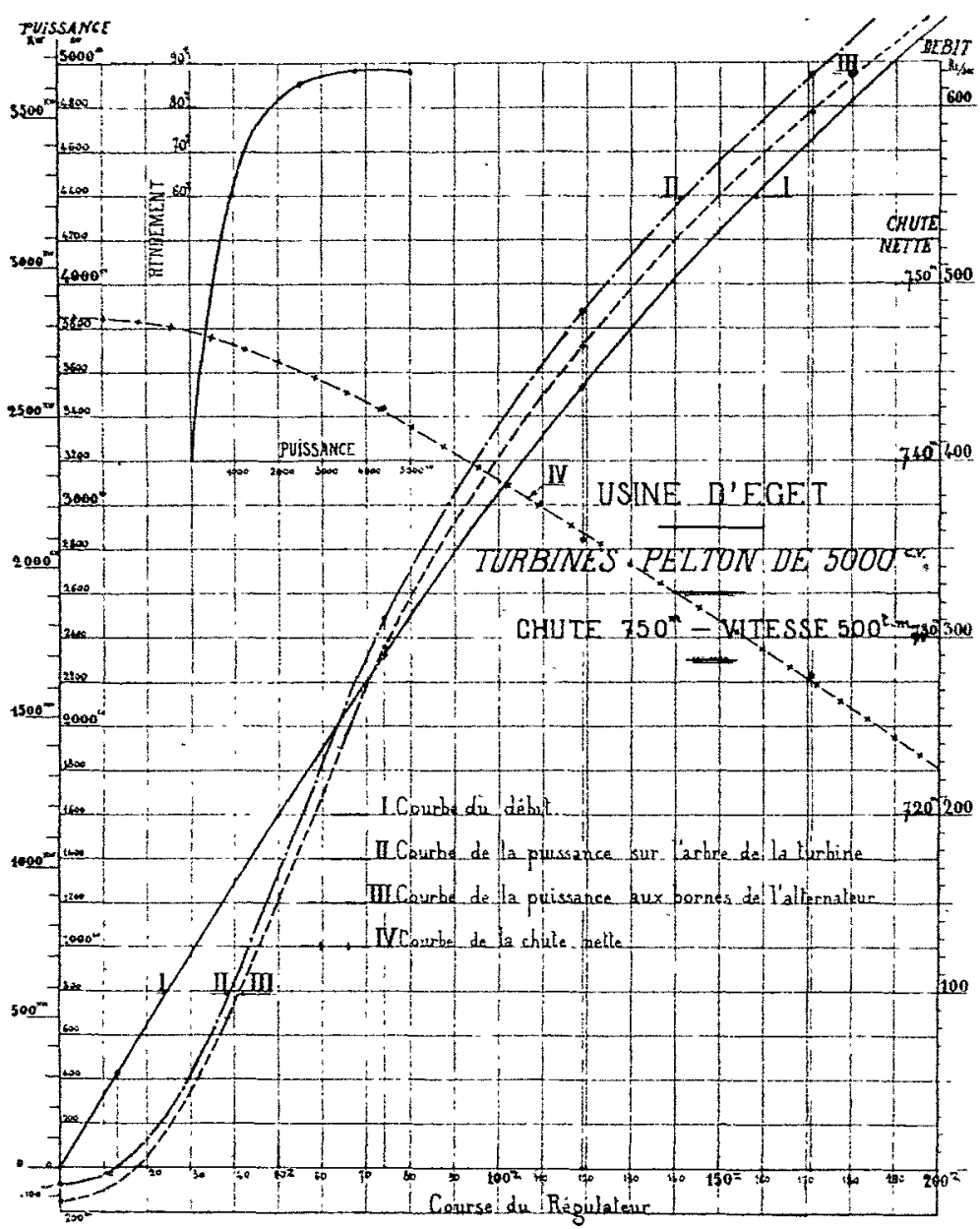

Fis 20

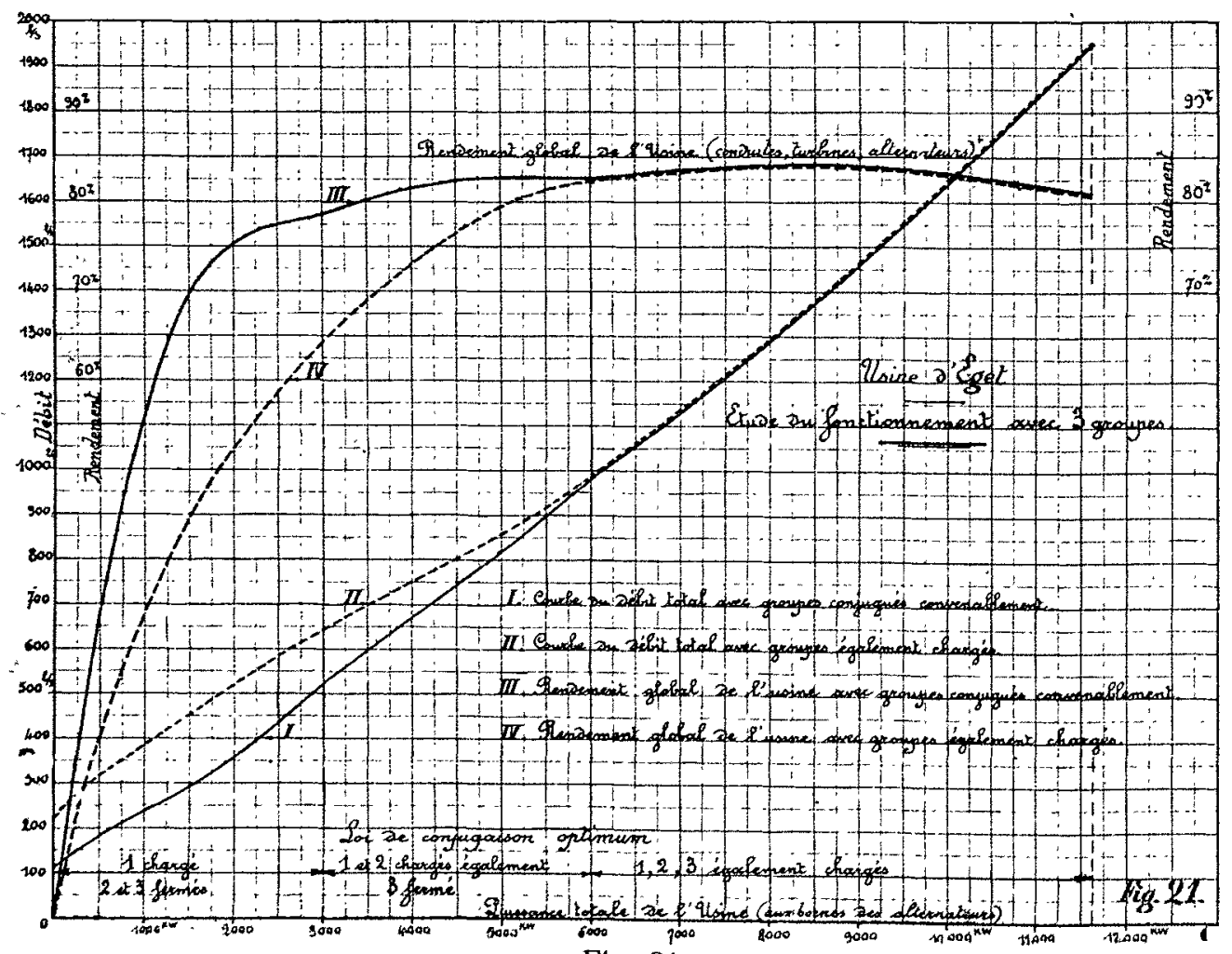

Fig. 21 nant ces valeurs à une chute nette constante, la chute nette niesurée est d'ailleurs portée sur le graphique.

Fn considerant les courbes I ct III de cette figure, donnant le débit et la puissance aux bornes de l'alternateur, nous pouvons dresser le tableau suivant, resumant les diverses condilions de fonctionnement des groupes supposés munis du dispositif précédemment décrit, assuranl la conjugaison optimum de ces groupes,

\begin{tabular}{|c|c|c|c|c|c|c|c|c|}
\hline \multirow{2}{*}{$\mid \begin{array}{c}\text { Débit } \\
\mathrm{Q}_{1}\end{array}$} & \multirow{2}{*}{$\begin{array}{c}\text { Débit } \\
Q\end{array}$} & \multirow{2}{*}{ Débit } & \multirow{2}{*}{$\begin{array}{l}\text { Debit } \\
\text { total }\end{array}$} & \multicolumn{4}{|c|}{ I'uissance aux bornes des alternateurs } & \\
\hline & & & & $P_{1}$ & $P_{*}$ & $P_{s}$ & I total & \\
\hline$\overline{1 / \text { sec. }}$ & $\overline{1 / \mathrm{sec}}$ & $\overline{1 / \mathrm{sec}}$ & $1_{i}$ sece. & KW & KW & KW & $\overline{\mathrm{KW}}$ & \\
\hline 116 & 0 & 0 & & +220 & -110 & -110 & 0 & 0 \\
\hline 200 & 0 & 0 & 200 & +880 & -110 & -110 & $\begin{array}{l}+660 \\
+\quad 6\end{array}$ & \\
\hline 300 & 0 & 0 & 300 & +1800 & -110 & -110 & +1580 & \\
\hline 400 & 0 & 0 & 400 & +2490 & -110 & -110 & +2270 & 77 \\
\hline 500 & 0 & 0 & 500 & +3080 & -110 & -110 & +2860 & 78 \\
\hline 600 & 0 & 0 & 600 & +3510 & -110 & -110 & +3290 & 76 \\
\hline 650 & 0 & 0 & 650 & +3870 & -110 & -110 & +3650 & 76 \\
\hline 325 & 325 & 0 & 650 & +2000 & $-\quad 0$ & -110 & +3890 & 81 \\
\hline 350 & 350 & 0 & 700 & +2150 & -+2150 & -110 & +4190 & 81 \\
\hline 400 & 400 & 0 & 800 & +2490 & +2490 & -110 & +4870 & 82 \\
\hline 500 & 500 & 0 & 1000 & +3070 & -3070 & -110 & +6030 & 82 \\
\hline 600 & 600 & 0 & 1200 & +3610 & +3610 & -110 & +7110 & 81 \\
\hline 650 & 650 & 0 & 1300 & +3870 & +3870 & -110 & +7630 & 80,5 \\
\hline 433 & 433 & 433 & 1300 & +2680 & +2680 & +2680 & +8040 & 84 \\
\hline 500 & 500 & 500 & 1500 & +3070 & +3070 & +3070 & +9210 & 84 \\
\hline 600 & 600 & 600 & 1800 & +3610 & +3610 & +3610 & +10830 & 81, \\
\hline 650 & 650 & 650 & 1950 & +3870 & $\frac{1}{1}-3870$ & +3870 & +11610 & \\
\hline
\end{tabular}

L'examen du tableau montre que le fonctionnement d'un slue groupe à pleine ouverture, les deux autres étant fermés, est un peu moins avantageux que le fonctionnement avec deux groujes à demi-charge, le troisième seul étant fermé. Cela tient aux pertes de charge dans les conduites (à Eget chaque turbine a sa conduite propre) dont l'importance aux grandes ouverture diminue sensiblement le rendement global de l'ensemble : conduite, turbine, alternateur.

Il faut donc ici mettre deux turbines en charge, à partir de 3.000 $\mathrm{KW}$, et trois turbines à partir de $6.000 \mathrm{KW}$, ce qui est facile, en disposant convenablement le distributeur commandé par le wattmètre qui assure la conjugaison des groupes.

La figure 21 , résumant cette étude, montre que pour la marche à $1 / 4$ de charge, le rendement global de l'installation, qui est de $62 \%$ avec les trois groupes à $1 / 4$ de charge, serait porté de $62 \%$ à $78 \%$ par une conjugaison convenable des charges des groupes, ce qui donnerait une économie de :

$$
\frac{78-62}{62}=25 \%
$$

sur le débit.

Ce résultat suffit à montrer que les mécanismes que nous venons d'étudier présenteraient un réel intérêt pratique.

\section{Conclusion}

Nous arrêterons ici cette étude dans laquelle nous avons seule- ment voulu fixer quelques problemes et esquisser rapidement leurs diverses solutions.

Nous espérons que les considérations qui précèdent, malgré leur caractère un peu spécial, pourront in téresser les techniciens de la Houille Blanche et, en attirant leur attention sur le rôle un peu méconnu des régulateurs dans l'économie générale des réseaux; contribueront a assurer une utilisation tonjours plus parfaite de nos installations hydro-electriques: 